\title{
Cuidar en la era tecnocientífica: De la finitud al superhombre
}

\section{Caring in the technoscientific era: From finitude to Overman}

\section{Cuidar na era tecnocientífica: da finitude ao super-homem}

\section{John Camilo García Uribe ${ }^{1}$}

\author{
${ }^{1}$ Enfermero profesional Universidad de Antioquia, maestrando en Bioética Universidad CES \\ Orcid:0000-0002-3810-5583. \\ Correspondencia: Medellín-Bello, Transversal 38aa\# 59a 231 (Colombia) \\ Correo electrónico de contacto: Johnc.garcia@udea.edu.co
}

Cómo citar este artículo: García-Uribe, J.C. (2021) Cuidar en la era tecnocientífica: De la finitud al superhombre Cultura de los Cuidados (Edición digital), 25(59). Recuperado de http://dx.doi.org/10.14198/cuid.2021.59.07

Recibido:02/11/2020 Aceptado: 10/01/2021

\section{RESUMEN}

El objetivo de este artículo de carácter reflexivo, es analizar la técnica y el cuidado como esencias humanas y como son su relaciones y efectos, en la contemporaneidad humanista enrutada hacia el posthumanismo. Metodología: La experiencia de cuidado en ámbitos altamente tecnificados, han creado cuestionamientos por el sentido y la esencia del cuidado en espacios tecnificados. A partir de estos dos grandes conceptos, se recorre un camino hermenéutico. Con referentes teóricos como Heiddeger, Nietzche y Mélich para buscar respuestas y nuevas preguntas en una contemporaneidad "dataista" y tecnificada, que anula el cuidado como esencia humana. Conclusión: Una solución ante la posible obsolescencia del cuidado, no es dejar de lado lo técnico, sino reavivar el cuidar del cuidado, resignificar el significado de la otredad que erróneamente es invisibilizada tras el telón de una obsolescencia programada y aparentemente necesaria. Cuidar del cuidado, es verdaderamente cuidar del otro y de esta casa común, es reconocer la finitud como orientación, como posibilidad, como una respuesta y una apertura al cuidado que permite cerrar brechas y abrir oportunidades. Es considerar lo técnico, no como un mecanismo emplazador-explotador, sino, por el contrario, cuidador.

Palabras Clave: Cuidado; técnica; humanismo; posthumanismo. 


\section{ABSTRACT}

The objective of this reflective article is to analyze technique and care as human essences and how their relationship and effects are in the humanistic contemporaneity routed towards posthumanism. Methodology: The experience of care in highly technical areas have created questions about the meaning and essence of care in technical spaces. From these two great concepts, a hermeneutical path is traveled. With theoretical references such as Heiddeger, Nietzche and Mélich to search for answers and new questions in a "dataist" and technified contemporaneity, which nullifies care as a human essence. Conclusion: A solution to the possible obsolescence of care is not to leave the technical aside, but to revive the care of care, to resignify the meaning of otherness that is mistakenly made invisible behind the curtain of a programmed and apparently necessary obsolescence. Caring for care is truly caring for the other and for this common home, it is recognizing finitude as orientation, as a possibility, as a response and an opening to care that allows closing gaps and opening opportunities. It is to consider the technical, not as a locator-exploiter mechanism, but, on the contrary, a caretaker.

Keywords: Caring; technique; Humanism; Posthumanism.

\section{RESUMO}

O objetivo deste artigo reflexivo é analisar a técnica e o cuidado como essências humanas e como suas relações e efeitos estão na contemporaneidade humanística direcionada ao pós-humanismo. Metodologia: A experiência do cuidado em áreas altamente técnicas, criou questões sobre o significado e a essência do cuidado em espaços técnicos. A partir desses dois grandes conceitos, é percorrido um caminho hermenêutico. Com referenciais teóricos como Heiddeger, Nietzche e Mélich, para buscar respostas e novas questões em uma "dataista" e contemporaneidade tecnificada, que anula o cuidado como essência humana.
Conclusão: Uma solução para a possível obsolescência do cuidado não é deixar o técnico de lado, mas reviver o cuidado, ressignificar o significado de alteridade que por engano se torna invisível por trás da cortina de uma obsolescência programada e aparentemente necessária. Cuidar é realmente cuidar do outro e deste lar comum, é reconhecer a finitude como orientação, como uma possibilidade, como uma resposta e uma abertura ao cuidado que permite fechar lacunas e abrir oportunidades. É considerar o técnico, não como um mecanismo localizador-explorador, mas, pelo contrário, um zelador.

\section{INTRODUCCIÓN}

El cuidado y la técnica son existenciarios del hombre, ambos son piezas fundamentales de los procesos de hominización y humanización. Ambos surgen de la condición humana de ser en el mundo, de estar arrojado (Heidegger, 2018) del extrañamiento frente al mundo (Esquirol, 2011), de su finitud (Melich, 2014), de la necesidad de otro (Levinas, 2012) y de su carencia. La técnica es la estrategia de la que se vale el hombre para sobrevivir adaptando el medio ambiente a sí mismo, mientras que los animales se adaptan al medio, lo técnico nos hace humanos (Montoya Suárez, 2008), y esa manera de adaptar el medio para la supervivencia y satisfacción de necesidades podría verse a groso como un cuidado, es decir la técnica como medio para el cuidado, o el cuidado como un gesto técnico, pero el cuidado no es esencialmente técnico. El cuidado es algo más, el cuidado como respuesta, como forma de ser en el mundo, precede y excede la existencia misma, desde antes de nacer se es cuidado, e incluso después de nacer se es cuidado, desde las patadas en el vientre, la respuesta frente al niño que llora al nacer, los cuidados después de morir, incluso los cuidados a los recuerdos e historias construidas, podría decirse de esta manera, que el cuidado precede y excede la técnica. Sin embargo, la actualidad técnificada pareciera terminar sobreponiéndose ante el cuidado, ambos son consustanciales, son rasgos esencialmente humanos, pero han tomado rumbos 
diferentes, a tal punto que uno termina subyugando al otro.

\section{ESTADO DE LA CUESTIÓN}

El origen fenomenológico del “cuidado" y la importancia del concepto de tiempo en la historia de la enfermería ya ha sido ampliamente abordado por otros autores (Siles González \& Solano Ruiz, 2007), donde abordan la equivalencia etimológica entre los términos Cura y Cuidado desde la propuesta teórica de Martin Heidegger a la que vez se analiza el cuidado a través desde la ontología y el Mito de Higinio en la obra Ser y Tiempo de Heidegger. La relación entre el concepto tiempo y el cuidado tanto como concepto como practica es también trabajado por estos autores(Siles González \& Solano Ruiz, 2007), diferenciando el tiempo cronológico "Crhonos" del tiempo que requiere cada cosa "Kairos", la dimensión temporal del cuidado implica tanto de Crhonos como de Kairos, en la medida que implica una construcción de una temporalidad determinada por la relación cuidador y sujeto de cuidado tal como es postulado por (Habran \& Battard, 2019) diferentes formas de producir relaciones de cuidado implican diferentes concepciones y orientaciones temporales, al considerar cómo diferentes formas de relaciones cuidador-cuidador pueden ayudar a construir el tiempo de manera diferente, pero también considerando el papel de las herramientas y otros actores, cuidar como expresión de un ser temporal, no puede ser mas que temporal, y esa temporalidad es construida, no son números llanos. Precisamente, por dicha asociación al tiempo, el cuidado no es ajeno al tiempo tecnificado del presente, de hecho, expresiones como el tiempo es oro, somo tiempo, no son una metáfora profunda, sino una fría lectura de la realidad, en la que a través de los avances tecnológicos se busca reducir los tiempos de producción, incluso los tiempos de cuidado (aquellos peligrosos, en los que el principio de precaución debería prevalecer, como con el desarrollo de una vacuna) (Singh, 2020), el tiempo para cuidar y por supuesto la construcción de tiempo entre sujeto de cuidado y cuidador.
La técnica no como herramienta, sino como significante o constructo social, es solo uno de los factores que podría influir en la deconstrucción del cuidado o la bien conocida deshumanización en salud(Arredondo-González \& SilesGonzález, 2009). En gran medida la técnica, es fruto de un paradigma científico positivista en la que existe una escisión entre el sujeto que investiga y el o lo investigado y a su vez está inmersa en un mundo mercantilizado y competitivo, donde la ganancia propia se sobrepone ante cualquier valor. Es en esta misma lógica de los tiempos actuales y venideros, donde los enfermeros y sujetos de cuidado hemos de vérnoslas.

\section{MÉTODOS}

La metodología, está fundamentada en dos pilares: La experiencia de cuidado en ámbitos altamente tecnificados que permite crear cuestionamientos por el sentido y la esencia del cuidado en espacios tecnificados. Desde la hermenéutica, , "que consiste ante todo en que uno puede considerar $\mathrm{y}$ reconsiderar lo que piensa su interlocutor, aunque no esté de acuerdo con él o ella , en la que se pone especial importancia al carácter móvil y finito de la existencia, donde la verdad y la forma de ver el mundo son siempre temporales” (de la Maza, 2005), desde los cuestionamientos y vacíos es posible construir, pero en mundo donde todo parece saberse o conocerse, pocas preguntas e interpretaciones surgen y con ello la normalización tiene lugar.

Esto, es precisamente una apuesta por trascender aquello que se ha instaurado de una manera dócil en la vida cotidiana y en el cuidado de enfermería. Es una exégesis y diálogo a través de postulados de la obra Ser y Tiempo de Heidegger, algunos axiomas de Nietzsche y una lógica interpretativa desde la filosofía de Mélich, a través de sus obras Ética compasión y Lógica de la crueldad, las cuales, aunque no parecen estar textualmente, están embebidas a lo largo y ancho de él. La insistencia en la finitud de la existencia y, por tanto, de la comprensión, pretende acentuar el alcance de todo conocimiento, en contraste con la pretensión de tener un conocimiento objetivo como el 
que persiguen las ciencias, como si fuera posible asentar su carácter definitivo por el mero recurso del método $y$, lo que es más importante, como si de ese modo fuera posible que el ser humano lograra una comprensión definitiva de sí mismo(Armando, 2004). Es desde esta lógica critica-argumentativa que se aborda el tema.

\section{DESARROLLO DEL TEMA}

\section{La técnica y el cuidado como esencia del ser en el mundo}

Para algunos, los primeros indicios de humanidad han sido los hallazgos arqueológicos de las herramientas utilizadas por nuestros antecesores, los homínidos, es decir la técnica (Harari, 2011). Para Ira Byock (2012), citando a Margaret Mead, los primeros signos de civilización no fueron anzuelos, ollas de barro o piedras de moler, sino un fémur roto que se había curado, en el reino animal si te rompes una pierna, mueres. No puedes huir del peligro, ir al rio a tomar algo o buscar comida. Eres carne de bestias que merodean. Ningún animal sobrevive a una pierna rota el tiempo suficiente para que el hueso sane. Un fémur roto que se ha curado es evidencia de que alguien se ha tomado el tiempo para quedarse con el que se cayó, ha vendado la herida, le ha llevado a un lugar seguro y le ha ayudado a recuperarse. ¿Cuál es el valor de establecer que fue lo primero si el cuidado o la técnica? Si ambos surgen desde la condición humana, si ambos contribuyeron a constituir la humanidad de hoy en día, ¿por qué termina prevaleciendo una sobre el otro? ¿Cuáles son los efectos a largo plazo? ¿porque habría de centrar el foco en que fue primero? Precisamente porque en la actualidad hay un descuido del cuidado y un dominio de lo técnico.

De acuerdo con Harari (2011), la prematuridad fisiológica al nacer fue una de las piezas claves en el proceso de hominización. Dado que contribuyó al desarrollo de las extraordinarias capacidades sociales de la humanidad. "Las madres solitarias apenas podían conseguir suficiente comida para su prole y para ellas al llevar consigo niños necesitados. Criar a los niños requería la ayuda constante de otros miembros de la familia y los vecinos. Para criar a un humano hace falta una tribu. Así, la evolución favoreció a los que eran capaces de crear lazos sociales fuertes (Harari, 2011). Pareciera entonces, que la condición de invalidez y prematuridad favoreció no solo el cuidado, sino la cooperación y el establecimiento de vínculos sociales para cooperar a mayor escala y no solo eso, esa misma prematuridad, le da la maleabilidad necesaria al ser humano para ser educado, para aprehender una amplia gama de habilidades y entre ellas la técnica.

Ahora bien, si analizamos la condición humana, en términos de la carencia biológica descrita por Gehlen (1987) como una prematuridad al nacer, con falta de pelo y protección natural frente a la intemperie, falta de órganos de ataque y formación corporal para huida”. Todo ello hace del hombre un ser dependiente al nacer, que depende de otro y de su cuidado. El no tener pelo y tener piel es un llamado al tacto del otro, a la cercanía, es un grito de auxilio, es un llamado para ser cuidado, en la cercanía surgen las relaciones de cuidado que favorecen pasar de la carencia a la abundancia. La carencia biológica de las crías no solo es un llamado para ser cuidado, también es el motor de respuesta para que el ser humano adulto haga frente y compense la situación de invalidez y carencia de sus crías(Fernández, 2018), de la carencia surge el cuidado y desde el cuidado se trasciende la carencia para llegar a la abundancia.

El cuidado es esencialmente humano y precede la técnica, citando a la fábula de Higinio y el mito de cuidado, expuesto por Heidegger (Ser y Tiempo, s. f.)

“Al atravesar Cuidado a un río, ve un gredoso barro, y cogiéndolo meditabunda lo comenzó a modelar. Mientras piensa en lo que hiciera, Júpiter se presenta. Cuidado pide le dé espíritu y fácilmente lo consigue. Como Cuidado quisiese darle su propio nombre, niégase Júpiter y exige se le ponga el suyo. Mientras ellos discuten, interviene también la Tierra pidiendo que su nombre sea dado a quien ella el cuerpo diera. Tomaron por juez a Saturno, y éste, equitativo, juzga: 'Tú, Júpiter, porque el espíritu le diste, en la muerte el espíritu y tú, 
Tierra, pues le diste el cuerpo, el cuerpo recibid, reténgalo Cuidado mientras viva, porque fue la primera en modelarlo. $\mathrm{Y}$ en cuanto a la disputa entre vosotros por el nombre, llámesele hombre, ya que del humus ha sido hecho”. (p. 198).

El hombre tiene su origen en el cuidado y es retenido por el mientras viva en el mundo, sin cuidado perecemos, sin cuidado el ser en el mundo no es. Sin una tierra (se entienden todos los elementos de la naturaleza) que le permita ser, y lo dote de consistencia, el ser en el mundo no puede llegar ser, somos tierra y volvemos a ella. Sin Saturno como tiempo, como aquel el juez implacable en el que todo tiene principio y fin, llegaríamos a ser, pero dejaríamos de ser. Pareciera de manera ontológica, que el ser en el mundo no es solo cuidado, somos tiempo, somos tierra y somos algo más allá de lo natural. No obstante, cabe preguntar, ¿Tiene la técnica asidero esta fábula? La técnica es de lo que se valió cuidado para hallar al ser en el mundo, para forjarlo y moldearlo a partir de la tierra y para mantenerlo en el mundo. Un mundo en el que el cuidado es la esencia del hombre, la técnica esta al servicio del cuidado, todo esta permeado por cuidado, todos los ámbitos técnicos, antes que técnicos, deben ser cuidados. Allí pudiera estar la subversión al gran problema de nuestra era tecnológica pero descuidada en tantos aspectos. Sin embargo, vale la pena ahondar en otros aspectos esencialmente humanos, que motivan la técnica y el cuidado.

El ser en el mundo, es un ser arrojado, carente y vulnerable, en medio de esa condición de vulnerabilidad, se la hace propio ocultar esa condición de vulnerabilidad (Torralba Roselló, 2013), para maquillar esa circunstancia vulnerable, de necesidad, de atención y de cuidado. En ese esconder el hombre se vale de la técnica, inicialmente como medida paliativa, para huir y solo olvidar, pero ahora en la contemporaneidad, la esencia de la técnica moderna es ostensiblemente diferente de la técnica antigua (cuidadora), y con ello los alcances, efectos esperados y no esperados han mutado, al punto que ya no solo es paliativa y cuidado, sino también curativa respecto a la condición de vulnerabilidad humana, para anularla desde todo punto de vista en una ruta que espera alcanzar la invulnerabilidad, la inmortalidad y la amortalidad. Porque para algunos, de acuerdo con Ayén (2015)“"estar atados a un sustrato biológico es malo, nos hace sufrir. Si el carácter humano pudiera darse en otro tipo de soportes que no fueran biológicos, habría una serie de limitaciones que no tendríamos". Mientras que, para otros, es la vulnerabilidad el motor y lo verdaderamente constituyente de lo humano.

Hoy en día, cada vez más, se busca anular la vulnerabilidad como condición humana, desde la muerte como fin de la existencia, hasta el dolor y el sufrimiento. Hoy se habla de la cura del envejecimiento (García-Barranquero, s. f.), y algunos más osados "afirman que hacia 2045, "llegaremos a la «muerte de la muerte», donde esta será opcional. Moriremos a causa de accidentes, pero nunca por muerte natural. Nos dirigimos hacia un futuro donde la muerte no tendrá dominio" (Cordeiro \& Wood, 2018)". No son solo libros, papers, y algunos científicos los interesados en esta causa, gigantes tecnológicos como Google, IBM y Microsoft, invierten millones de dólares en investigación y desarrollo en la lucha contra la muerte y el envejecimiento(Espallargas, 2019), y un movimiento filosófico se ha desarrollado entorno al posthumanismo. De manera superficial, podría parecer un capítulo de un libro de ciencia ficción, pero la realidad, deja en el aire algunas señales; de que todo esto puede materializarse a través de grandes avances en nanotecnología, biotecnología e inteligencia artificial. De este modo, los tiempos actuales son tiempos, en los que la esperanza y los ojos están puestos en la tecnociencia, como solución a nuestra condición de vulnerabilidad y como la vía para la supervivencia de la especie, sea en este $o$ en otro planeta.

Este volcamiento a la tecnociencia tiene efectos a toda escala, en la medida que la esencia de la técnica moderna, radica en la técnica como estructura de emplazamiento (“Ge-stell”) descrito por (Heidegger, 1994), de modo que se ve la naturaleza y el hombre como recurso, recurso explotable del que no habría que tener cuidado alguno. El 
superhombre contemporáneo está enmarcado en un modelo egocéntrico y tecnocrático, que parece hacerle honor a la teoría evolucionista, en la que las especies mejor adaptadas en medio de recursos finitos son las que más sobreviven, de manera que hay una pugna mas no una cooperación, ni mucho menos un cuidado por el otro, sino por el contrario un interés egocéntrico, en el que la tecnociencia tiene la respuesta a todos los problemas, desde el terrorismo, el hambre, el cambio climático, la pobreza y el desempleo, hasta la muerte misma. Sin embargo, en un campo en el que en el que la compasión del buen samaritano es olvidada tras la muerte de Dios (Nietzsche, 2001), y el cuidado queda relegado, la tecnología carece de la profundidad y el alcance necesarios para la continuidad de la vida en la tierra y del hombre como especie. Lo mas neurálgico no es ello, sino ver como el cuidado como técnica no surge como alternativa ante las crisis: desde las sanitarias-hasta las ambientales. Sino por el contrario, parece una pesadilla del eterno retorno Nietzscheano, en el que se recurre a lo mismo (lo técnico), con la excusa de cuidar para vivir de la mejor manera posible, una vida enmarcada en la temporalidad, el problema es que esa vida que se pretende cuidar y repetir no puede estar aislada, pues como seres abiertos hacemos parte de un sistema, donde todo esta interconectado tanto de manera física como fenomenológica.

En estos tiempos, la técnica deja de ser un instrumento, un hacer para cuidar y formar al hombre para ser una tecnociencia endiosada, en la que surge la posibilidad de una solución ante la muerte y/o la posibilidad de reemplazar aquello que se dañe y perece con vasta facilidad. Con lo cual la necesidad de cuidar se hace superflua, innecesaria, un mero aditamento, llegando al punto, que nos preguntaremos el para qué cuidar si nada muere, o incluso, un punto cronológicamente más cercano, más próximo, en el que preguntamos para qué cuidar algo si lo puedo reemplazar por algo mejor. De esta manera, la tecnociencia es la luz que ilumina lo oscuro, es un Dios cada vez más explícito, omnipresente y omnipotente, cada vez más capaz de reemplazar a Cuidado, pero también capaz de vencer a Júpiter como dador de vida, a Tierra como lugar de origen y morada y a Saturno como juez implacable de la temporaneidad.

\section{La muerte de Dios y la coronación de la Tecnociencia}

Las ficciones, la capacidad de pensar sobre cosas que realmente no existen, de manera individual y colectiva ha "permitido urdir mitos comunes tales como la historia bíblica de la creación, los mitos del tiempo del sueño de los aborígenes australianos, y los mitos nacionalistas de los estados modernos. Dichos mitos confirieron a los sapiens la capacidad sin precedentes de cooperar flexiblemente en gran número"(Harari, 2011). El mito o los mitos comunes de una sociedad favorecen y moldean un sinnúmero de interrelaciones, que trascienden la cooperación misma y generan otros mitos. No es la intención de este escrito hablar sobre los diferentes mitos que han surgido a lo largo de la historia del hombre, pero es necesario, hacer alusión al mito de Dios, o al menos a la creencia en Dios, como modelo de visión del mundo y con ello, de comportamiento y de cooperación , para luego analizar las implicaciones de "la muerte de Dios", en las que otra creencia colectiva permea a cada persona y a cada rincón del planeta, el mito de la tecnociencia.

La muerte de Dios no es una expresión literal, de manera literal sería un sinsentido en la medida que Dios no muere. Dicha expresión no se refiere a un Dios como ser viviente, sino a la creencia en un Dios, un algo, que dotaba de sentido la existencia y con ello daba paso a una moral universalmente controladora. La muerte de Dios (Nietzsche, 2003) es una venda autoimpuesta, que cae de los ojos para contemplar la verdad, pero pareciera que necesitamos de una venda, de la dificultad, de algo que tenga que ser des-ocultado y revelado, el traer ahí, el hacer presente es un hacer humano. La ausencia de Dios dejaría un vacío abismal en la existencia del hombre, una carencia más. El hombre, el asesino, podría estar asesinando su propia humanidad, o creando una nueva, en la medida que algo tiene que necesariamente reemplazar a Dios, un nuevo sentido, un 
nuevo mito y con ello tal vez una nueva humanidad. Mas se demora una Deidad en irse que otra en llegar, muerto Dios, endiosada la tecnociencia, esta, es ahora la esencia dominante del ser en el mundo, a través del Ge-stell (Heidegger, s. f.), en el que se dispone de recursos, esta cosifica la realidad, la vida y los seres, la esencia pasa a ser una nimiedad y la utilidad es la medida de todo lo explotable.

Muerto Dios, el superhombre ve en la tecnociencia, mas que un camino, un vuelo directo a al Olimpo, a las cosechas de ambrosia. No significa que muerto Dios no hay religiosidad, al contrario, el fervor tecnocrático mueve no solo cuerpos, sino subjetividades e intersubjetividades. La tecnociencia como deidad, es un proyectil magnético que sale disparado y a medida que avanza subvierte todo a su paso, con lleva la humanidad a la muerte. Muerto Dios, muerto el hombre, pero la muerte no es el fin, sino la transformación de lo humano es el surgir del superhombre, no como un hombre mejorado, sino un nuevo hombre. Un hombre deificado, inmortal, con dominio sobre la vida y la muerte, un hombre descuidadamente omnipotente. El advenimiento de un nuevo tipo de ser humano, un posthumano, el ultra hombre, aquella especie que reemplaza al sapiens, que sabe vivir sin Dios, sin centro, es romper la idea de que necesitamos un fondo, es romper todos los esquemas, es ser Dios, un ser que no necesita sentido para existir y por supuesto no se cuida, no cuida, y no requiere cuidados.

La Presencia de Dios además de sustentar y dar rumbo a la existencia del ser en el mundo, a travesaba la humanidad en su esencia, por ello las consecuencias de su muerte, de su ausencia, de su reemplazo y de finalmente su futilidad son a todo nivel, desde el sistema moral y de valores, hasta ámbitos en los que el cuidado, como ontología y como práctica, parecía ser eminentemente dominante y esencial, pero que finalmente ha quedado supeditado a un sistema tecnocientífico, emplazador, que sataniza la compasión y consiguientemente el cuidado por lo otro y el otro.

Cuidado de la salud y humanismo
Muerto Dios, se ha endiosado lo humano y con ello el humanismo surge como respuesta a la ausencia divina, como la nueva religión que moldea la relaciones del presente. Con esta nueva cosmogonía dominante, se transfixian todas las microesferas humanas, superfluas y esenciales. El cuidado de la salud, no se escapa de ello. La muerte de Dios trae consecuencias para cuidadores y sujetos de cuidado. Salud y enfermedad dejan de ser regalos o castigos divinos, para estar ligados a factores genéticos, epigenéticos, ambientales y estilos de vida. De esta manera, el estar saludable o enfermero se debe una interrelación de factores heredados, individuales, y sociales, enfermedades como el Cancer dejan de ser un castigo, para estar ligadas a genes cancerígenos, a exposiciones ambientales o hábitos como el tabaquismo. Pero de esta misma forma, la búsqueda de la salud ya no se espera provenga de poderes divinos, sino de acciones humanas (medicamentos, biotecnología, nanotecnología, entre otros). Los profesionales de la salud han dejado de ser enviados mesiánicos con poderes sobrenaturales o divinos para ser vistos como un actor más del modelo de mercado, del que ya no se espera un cuidado, sino al que se le exige un cuidado con "calidad". Los pacientes, ya no son tan pacientes, ahora son activos y algunas veces reactivos.

El cuidado de la salud, del ambiente y del otro, ha salido del enfoque orientador del actuar humano. ¿para que cuidar algo si se puede reemplazar por algo mejor? ¿para que mantener estilos de vida saludables, si la tecnología lo soluciona todo? Pero rara vez nos preguntamos, ¿cuál es el costo de todo esto? Costo social, familiar, ambiental y personal. Esta manera de solucionar produciendo y no cuidando, descuidando y malgastando, favorece la aparición de enfermedades obesidad, vigorexia, la depresión, el trastorno por déficit de atención con hiperactividad (TDAH), el trastorno límite de la personalidad (TLP) o el síndrome de desgaste ocupacional (SDO), enfermedades hipercatatonicas, que requieren respuestas acordes al sistema de producción, es decir, la solución no es parar, la solución es producir nuevos medicamentos, un curso de coaching o la 
implementación de nuevos desarrollos tecnológicos.

La sacralidad de la vida humana ya no proviene por el origen divino, sino por una superioridad especista, que ha determinado el poder y dominio sobre las demás especies y sobre el planeta entero. La sensación de superioridad y grandeza que poseemos gracias a la selección natural del más fuerte y mejor adaptado, parece ser suficiente para contemplar lo otro, no como otro necesario para mí, sino como un recurso del que se dispone y se explota. Así, El actuar humano $\mathrm{y}$ sus consecuencias deletéreas sobre otras especies y el ambiente, se tienden a justificar errónea y vilmente. Con este mismo enfoque, se ha sacralizado el valor de la vida humana sobre cualquier otra forma de vida y de igual forma parecieran existir vidas humanas más valiosas que otras. La sensación de la igualdad del valor de la vida es una mera apariencia, puesto que aquellos con más poder gozan de mayores cuidados. Y lo único igual para todos los seres vivos, está a punto de cambiar, de cambiar para algunos cuantos poderosos. Pues grandes esfuerzos se hacen para no solo mantener con vida a toda costa los seres humanos, sino que también se pretende anular la muerte o trasladar la "vida" a entidades no perecederas. En este camino tortuoso de humanismo a posthumanismo, la tecnociencia cobra cada vez mas importancia, y parece zanjar más distancias entre el cuidado y producción. Sin embargo, el cuidado contiene la técnica, son consustancialmente humanos y es menester recordar y reavivar su importancia, para no perder la esencia humana.

\section{El cuidado de la salud en la era tecnológica}

Las nuevas tecnologías resuelven grandes problemas en el ámbito de la salud, ahora a través de avances de la tecnociencia es posible tratar y curar enfermedades que, antes incluso, eran desconocidas, gracias a marcapasos, cardiodesfribiladores implantables, monitores avanzados de signos vitales, biofármacos, prótesis artificiales, quimioterapia y un sinnúmero de scores y protocolos de atención basados en la evidencia. Pero grandes avances, implican nuevos retos, nuevos problemas (Domingo, 2013). Desde el aumento significativo de la esperanza de vida y con ello de la población, la toma de decisiones frente a la vida y la muerte, hasta el acto de cuidado. Un cuidado cada vez más descuidado en su esencia y en la práctica, en el que pareciera que sin protocolos basados en la evidencia y sin aparatos tecnológicos este no tuviera lugar alguno, en el que importa más el monitor que el paciente, los signos se sobreponen a los síntomas, y los protocolos en muchos casos a las necesidades del paciente. A raíz de ello, entre un pleonasmo (cuidado humanizado) y un oxímoron (deshumanización del cuidado), surgen discursos un tanto llamativos, pero que desvían la atención del foco del problema, un olvido de la esencia del cuidado.

Así como en la vida cotidiana la televisión nos atonta, nos aturde de tal forma que quedamos como prendados de ella. "Con un efecto entre mágico y maléfico es obra, creo, del exceso de la luz que con su intensidad nos toma. Mismo efecto que produce en los insectos, y aun en los grandes animales” (Sabato, 2000). De esa misma manera las pantallas de monitores y maquinas destinados al cuidado de la salud nos invaden y domestican, a tal punto que no se mira al paciente sino el monitor, el monitor siempre tiene la razón, y arroja más y mejores "datos" que el paciente. En un mundo en que los datos y el flujo de datos son el objeto de mayor valor, el cuidado se ciñe a los mejores datos obtenibles, la evidencia científica y las pantallas de monitores, dejando de lado aquellos datos erróneamente clasificados como inservibles, la subjetividad del otro.

En la sociedad de los datos, "no vemos lo que no tiene la iluminación de la pantalla, ni oímos lo que no llega a nosotros cargado de decibeles, ni olemos perfumes. Ya ni las flores los tienen” (Sabato, 2000) ahora cuesta tanto levantarse ante el llamado del otro, porque implica una desconexión de la red de datos, los oídos son sordos antes las voces humanas, pero están ávidos por escuchar las alarmas y los pitidos de las maquinas. De esta misma forma, se ha modelado el enfermar y recuperarse y con ello el cuidado mismo, ahora pululan los 
blogs y consultas online a través de pantallas de celular y ordenadores, en las que, en algunos casos de manera superficial, se pretende dar soluciones a problemas tan profundos, que un sistema de datos binarios es incapaz de transmitir. Es el mismo dataismo quien reduce a categorías, a números y a estadísticas la identidad del enfermo y del que cuida.

\section{CONCLUSIÓN}

En un escenario como este, hablar de deshumanización del cuidado, hace pensar que es posible cuidar de manera deshumanizada, mientras que humanizar el cuidado carece de especificidad, pues son tantas las características humanas, que bien, podría ser tecnificar el cuidado o permearlo de compasión. Por lo que una acepción más acorde y plausible, seria cuidar del cuidado. Cuidar del cuidado en un mundo tecnológico, humanista y capitalista. Para ello se requiere, explotar "el único recurso que con su uso no se acaba, sino que se expande: el conocimiento"(Harari, 2016). El conocimiento no para producir, sino para cuidar, un conocimiento que no dará frutos instantáneos, pero si tardíos y duraderos. El imperativo del conocimiento para cuidar abarca desde los instrumentos, técnicas y nuevas tecnologías, hasta el conocimiento del otro y de las interrelaciones que favorecen y permiten la vida en y del planeta. El conocimiento ilumina lo oscuro, nos saca de la caverna para romper el velo del ego, del ensimismamiento, del endiosamiento y del estado catatónico de hiperproducción y consumo, en el que la técnica anula el cuidado. El conocimiento para parar y cuestionar y con ello responder desde el cuidar. Para ello, la solución no es dejar de lado lo técnico, sino reavivar el cuidar del cuidado, resignificar el significado de la otredad que erróneamente es invisibilizada tras el telón de una obsolescencia programada y aparentemente necesaria. Cuidar del cuidado, es verdaderamente cuidar del otro y de esta casa común, es reconocer la finitud como orientación, como posibilidad, como una respuesta y una apertura al cuidado que permite cerrar brechas y abrir oportunidades, mientras que gastar y descuidar con la esperanza en la muerte de la muerte abre brechas y quita oportunidades.

Es necesario cuidar del cuidado, tanto en el lenguaje como en la práctica implica recurrir a un cuidado desde aquella raíz latina "cogitare" (Corominas \& Pascual, 2010) “cogito" pensamiento, como posibilidad de hacer un espacio en la mente propia para el otro, un espacio que es ante todo posibilidad de ser con el otro, para el otro y en esa posibilidad de ser se constituye la propia existencia, el cuidado como pensamiento es reconocer la finitud y la interdependencia, es una apuesta por la compasión, el com-partir y el con-vivir (Mélich, 2013), todas ellas, como relaciones duales requieren de hacer un espacio para el otro, en mente, en tiempo y cuerpo, para construir, trascender y evitar un futuro apocalíptico, des-cuidado, sin posibilidad a pausas, preguntas, diálogos, relaciones, a causa de un cuidado descuidado. sobrevivirán?

$¿$ ¿n un mundo sin cuidado quienes

\section{BIBLIOGRAFÍA}

Armando, L. (2004). P U E R T O S La hermenéutica. 5.

Arredondo-González, C. P., \& SilesGonzález, J. (2009). Tecnología y Humanización de los Cuidados: Una mirada desde la Teoría de las Relaciones Interpersonales. Index de Enfermería, 18(1), 32-36.

Ayén, X. (2015). Vamos a ser inmortales. Recuperado de https://www.lavanguardia.com/cultura/2015 0809/54435718128/vamos-ser-

inmortales.html

Byock, I. (2012). The best care possible: A physician's quest to transform care through the end of life (1.a ed.). NY: Avery Pub Group

Cordeiro, J. L., \& Wood, D. (2018). La muerte de la muerte (Vol. 1). Bilbao: Deusto. 
Corominas, J., \& Pascual, J. M. (2010). Diccionario crítico etimológico castellano e hispánico (CE-F. 1. ed, 7. reimpr, Vol. 2). Madrid: Gredos.

de la Maza, L. M. (2005). Fundamentos de la filosofía hermenéutica: Heidegger y Gadamer. Teología y vida, 46(1-2). Recuperado de https://doi.org/10.4067/S004934492005000100006

Domingo, A. (2013). El arte de cuidar. Atender, dialogar y responder (1.a ed.). Madird: Rialp..

Espallargas, A. (2019). Proyecto Gilgamesh: Objetivo, curar la muerte para 2045. GQ España. Recuperado de https://www.revistagq.com/cuidados/articul os/proyecto-gilgamesh-inmortalidadhumana-2019/32876

Esquirol, J. M. (2011). Los Filósofos contemporáneos y la técnica: De Ortega a Sloterdijk (Primera edición). Madrid: Gedisa.

Fernández, M. A. (2018). Reflexiones en torno a la idea del hombre como ser carencial: Ortega, Gehlen y Sloterdijk. Thémata Revista de Filosofía, 58, 119-136.

García-Barranquero, P. (2016.). Aportes científicos y filosóficos del pensamiento de Aubrey de Grey. Gazeta de Antropología, 32(2), 01 Recueprado de http://hdl.handle.net/10481/43304 12.

Gehlen, A. (1987). El hombre: Su naturaleza y su lugar en el mundo. Madrid: Sigueme.

Habran, Y., \& Battard, N. (2019). Caring for or caring with? Production of different caring relationships and the construction of time. Social Science \& Medicine, 233, 78-86.

Harari, Y. N. (2011). Sapiens: De animales a dioses. Madrid: Debate.

Harari, Y. N. (2016). Homo Deus: Breve historia del mañana. Madrid: Penguin Random House Grupo Editorial España.

Heidegger, M. (s. f.). La pregunta por la técnica. Revista de Filosofía. Recuperado de file:///C:/Users/USUARIO-
PC/Downloads/45002-1-158744-1-10-

20170118.pdf

Heidegger, M. (1994). Conferencias y artículos (E. Barjau, Trad.). Barcelona: Ediciones Serbal.

Heidegger, M. (2018). Ser y tiempo (J. E. Rivera, Trad.; 3a ed.). Madrid: Trotta.

Levinas, E. (2012). Totalidad $e$ infinito.Madrid: Sígueme.

Mélich, J. C. (2013). Ética de la compasión. Barcelona: Herder.

Melich, J. C. (2014). Lógica de la crueldad. Barcelona: Herder.

Montoya Suárez, O. (2008). De la Techne Griega a la técnica Occidental Moderna. Scientia et Technica Año XIV, 39.

Nietzsche, F. (2001). La Gaya Ciencia. Madrid: Akal.

Nietzsche, F. (2003). Así habló Zaratustra: Un libro para todos y para ninguno. Madrid: Alianza.

Sábato, E. (2000). La resistencia. Barcelona: Seix Barral.

Siles González, J., \& Solano Ruiz, M. C. (2007). El origen fenomenológico del “cuidado" y la importancia del concepto de tiempo en la historia de la enfermería. Cultura de los Cuidados, 21, 19-27. Recuperado de https://doi.org/10.14198/cuid.2007.21.04

Singh, J. A. (2020). COVID-19 vaccine trials: Duty of care and standard of prevention considerations. Vaccine, 38(48), 7578-7580.

https://doi.org/10.1016/j.vaccine.2020.10.0 12

Torralba Roselló, F. (2013). Hacia una antropología de la vulnerabilidad. Revista Forma, 2. Recuperado de https://www.academia.edu/31666084/HACI A_UNA_ANTROPOLOG\%C3\%8DA_DE _LA_VULNERABILIDAD 